\title{
Midterm Results after Open versus Arthroscopic Transosseous Repair for Foveal Tears of the Triangular Fibrocartilage Complex
}

\author{
Yukio Abe, MD, PhD ${ }^{1} \quad$ Kenzo Fujii, MD ${ }^{1}$ Takeyoshi Fujisawa, MD ${ }^{1}$ \\ ${ }^{1}$ Department of Orthopaedic Surgery, Saiseikai Shimonoseki General \\ Hospital, Yamaguchi, Japan \\ Address for correspondence Yukio Abe, MD, PhD, Department of \\ Orthopaedic Surgery, Saiseikai Shimonoseki General Hospital, \\ Yamaguchi 759-6603, Japan \\ J Wrist Surg 2018;7:292-297. \\ (e-mail: handsurgeonabe@jcom.home.ne.jp).
}

\begin{abstract}
Purpose Various surgical procedures for foveal tears of the triangular fibrocartilage complex (TFCC) have been reported, and the procedures can be grossly divided into open and arthroscopic repair. The surgical results of both procedures were compared. Materials and Methods Twenty-nine patients underwent repair of a TFCC foveal tear. The 13 men and 16 women were in the age range of 14 to 72 years (average age, 30 years). Five patients had a history of distal radius fractures that healed uneventfully with nonoperative treatment. The mean duration of symptoms before surgery was 7.1 months. The procedure for repair consisted of 8 open repairs and 21 arthroscopic repairs. In both procedures, the TFCC was repaired transosseously to the ulna. The mean follow-up period was 34.4 (range, 24-70) months. The patients' pain, range of motion (ROM), grasping power, ulnar head instability, Disabilities of the Arm, Shoulder and Hand questionnaire (DASH), and Mayo modified wrist score (MMWS) were evaluated. The operating time was also compared.

Results There were no significant differences between the groups in pain, ROM, grasping power, ulnar head instability, and DASH. The MMWS was excellent in 8 patients in the open repair group, with 18 excellent and 3 good in the arthroscopic

\author{
Keywords \\ - wrist \\ - arthroscopy \\ - triangular \\ fibrocartilage \\ complex \\ - fovea
} repair group. The mean operating time was significantly shorter for arthroscopic repair than for open repair.

Conclusion Satisfactory outcomes were achieved for both open and arthroscopic repair techniques in the midterm. If a surgeon becomes familiar with the arthroscopic repair, the arthroscopic technique would be more feasible than the open repair in terms of technical facility and shortening of the operating time.

Level of Evidence Level III, therapeutic study.
\end{abstract}

The triangular fibrocartilage complex (TFCC) is a well-known structure that acts as a stabilizer of the distal radioulnar joint (DRUJ) and a shock absorber of the ulnocarpal joint. Recent anatomical studies have proven that the distal radioulnar ligament consists of superficial and deep bundles, which attach on the fovea and provides DRUJ stability. ${ }^{1-3}$ Haugstved et al demonstrated that the deep ligaments provide greater

received

August 31, 2017

accepted after revision

March 6, 2018

published online

April 10, 2018 stability of the DRUJ than the superficial ligaments in a biomechanical study. ${ }^{4}$ This indicates that disruption of the TFCC at the fovea insertion could lead to DRUJ instability, resulting in disability in daily living. In this situation, a foveal tear might be repaired. Several procedures have been recommended to repair a foveal tear, and they can be divided into open and arthroscopic repairs. However, there have been few
Copyright $\odot 2018$ by Thieme Medical Publishers, Inc., 333 Seventh Avenue, New York, NY 10001, USA. Tel: +1(212) 584-4662.
DOI https://doi.org/ 10.1055/s-0038-1641720. ISSN 2163-3916. 
reports that have compared the effectiveness of the open repair and the arthroscopic repair. ${ }^{5-7}$ The purpose of this study was to compare open with arthroscopic repair of foveal tears of the TFCC.

\section{Materials and Methods}

This was a retrospective study of a group of patients who had complained disability of the wrist and were found to have a foveal TFCC tear at the time of wrist arthroscopy. Informed consent was obtained from all patients, and the study protocol adhered to the ethical guidelines of the 1975 Declaration of Helsinki. This clinical investigation was conducted with the approval of our institutional review board. The preoperative findings were recorded following a retrospective record review.

Our indication for repair of a TFCC foveal tear was symptomatic DRUJ instability that had not responded to nonsurgical treatment for over 3 months. Therefore, in this study, the patients with a foveal tear associated with a fresh distal radius fracture (DRF) were excluded. The patients who had concomitant scapholunate ligament (SL) injury were also excluded. Furthermore, the patients who had foveal tear with an ulnar positive variance of more than $+1 \mathrm{~mm}$ and were initially treated with ulnar shortening osteotomy were also excluded.

Since December 2004 to January 2014, 42 wrists of 42 patients with a TFCC foveal tear were treated surgically. These included 5 patients with acute DRF, 1 patient associated with SL injury, and 7 patients treated with ulnar shortening osteotomy. Thus, 29 wrists of 29 patients with a TFCC foveal tear treated surgically were investigated. There were 13 men and 16 women, 14 right and 15 left wrists, and 16 dominant and 13 nondominant hands. The mean age of the patients was 30 (range, 14-72) years. Sixteen patients suffered the injury during sports activities, and 12 patients suffered the injury during working, by a fall, or twisting the wrist. One patient could not remember the clear history of wrist trauma. Five patients had a previous history of DRF that had healed uneventfully with normal alignment by cast immobilization.

The first eight patients between December 2004 and October 2008 underwent open repair (group 0). Twentyone patients between November 2008 and January 2014 were repaired arthroscopically (group A). The mean duration of symptoms before surgery was 7.1 months, ranging from 3 to 20 months. The follow-up period ranged from 24 to 70 months, with an average of 34.4 months. The age and duration of symptoms before surgery in each group are represented in - Table 1 .

\section{Clinical and Radiological Evaluation}

All patients complained of ulnar-sided wrist pain with wrist extension and forearm rotation. All patients also felt ulnar head instability during forceful forearm rotation. Some specific physical examinations for the foveal tear were examined. A positive fovea sign represented ulnar-sided wrist tenderness in the ulnar fovea. ${ }^{8}$ The ulnar head ballottement test was examined by the piano key sign with neutral forearm rotation and $90^{\circ}$ flexion of the elbow, holding the radius and the carpal bones. Obvious palmar and dorsal ulnar head instability compared with the contralateral wrist was diagnosed as positive in this test. All patients underwent a radiographic evaluation including neutral rotation posteroanterior and lateral X-rays, and $1.5 \mathrm{~T}$ coronal plane magnetic resonance imaging (MRI). On the X-rays, none represented ulnar styloid nonunion or DRUJ arthrosis. The mean ulnar variance was $0.7 \mathrm{~mm}(-3.0$ to $+0.3 \mathrm{~mm})$. Three patients showed a distended DRUJ joint over $1 \mathrm{~mm}$ compared with the contralateral $\mathrm{X}$-ray. One patient showed a small fragment just distal to the fovea. On MRI, 18 patients showed lack of continuity of TFCC at the fovea, whereas 11 patients seemed to demonstrate continuity at the fovea on MRI. These were graded by the agreement of two hand surgeons including the first author (Y.A.). All patients underwent an initial trial of conservative treatment,

Table 1 Patients' demographics and preoperative data in each group

\begin{tabular}{|c|c|c|c|c|}
\hline & $\begin{array}{l}\text { Group O } \\
(n=8)\end{array}$ & Maximum-Minimum & $\begin{array}{l}\text { Group A } \\
(n=21)\end{array}$ & Maximum-Minimum \\
\hline & Mean & & Mean & \\
\hline Age & 22 & $14-40$ & 34 & $14-72$ \\
\hline Period from onset to surgery (mo) & 11 & $3-20$ & 8.5 & $3-20$ \\
\hline Ulnar variance $(\mathrm{mm})$ & -0.6 & -2.5 to +0.3 & -0.7 & -3.0 to +0.3 \\
\hline Atzei's classification & $\begin{array}{l}\text { Class 2: } 4 \\
\text { Class 3: } 4\end{array}$ & & $\begin{array}{l}\text { Class 2: } 6 \\
\text { Class 3: } 15\end{array}$ & \\
\hline NRS & 10 & Only 10 & 10 & Only 10 \\
\hline Extension $\left({ }^{\circ}\right)$ & 71.7 & $64-80$ & 72.6 & $54-86$ \\
\hline Flexion $\left({ }^{\circ}\right)$ & 61.0 & $48-84$ & 59.6 & $45-81$ \\
\hline Pronation $\left({ }^{\circ}\right)$ & 83.3 & $70-90$ & 81.3 & $60-90$ \\
\hline Supination $\left({ }^{\circ}\right)$ & 89.1 & $75-90$ & 86.9 & $45-90$ \\
\hline Grip strength (\%) & 81.6 & $38-91$ & 80.2 & $38-100$ \\
\hline
\end{tabular}

Abbreviation: NRS, numerical rating scale. 
such as cast immobilization, splinting, and administration of nonsteroidal anti-inflammatory drugs, all of which failed.

All patients were assessed with wrist arthroscopy by single surgeon (Y.A.) including radiocarpal (RC) and DRUJ arthroscopy, and they were confirmed to have a foveal tear. According to Atzei's classification, ${ }^{9}$ there were 4 Class 2 (repairable complete tear) and 4 Class 3 (repairable proximal tear) in group 0 , and there were 6 Class 2 and 15 Class 3 in group A. Eleven patients who demonstrated continuity on MRI had fragile scar tissue at the fovea. To ensure interobserver reliability of the arthroscopic examinations, the foveal tear was confirmed by the first author and a scrub doctor intraoperatively, and reconfirmed by another surgeon who did not participate in the surgery through photographs postoperatively. These surgeons were qualified by our National Orthopaedic Association, and all surgeons agreed with the diagnosis of the foveal tear.

\section{Preoperative Data}

A fovea sign and ulnar head ballottement test were positive in all patients. The mean ulnar variance was $-0.6 \mathrm{~mm}(-2.5 \mathrm{~mm}$ to $+0.3 \mathrm{~mm})$ in group $0,-0.7 \mathrm{~mm}(-3.0 \mathrm{~mm}$ to $+0.3 \mathrm{~mm})$ in group A. Preoperative pain was scored as 10 in all patients with numerical rating scale (NRS). The mean extension of the wrist was $71.7^{\circ}$ (range: $64^{\circ}-80^{\circ}$ ), and mean flexion was $61.0^{\circ}$ (range: $48^{\circ}-84^{\circ}$ ) in group 0 ; the mean extension was $72.6^{\circ}$ (range: $54^{\circ}-86^{\circ}$ ), and the mean flexion was $59.6^{\circ}$ (range: $45^{\circ}-81^{\circ}$ ) in group A. The mean pronation of the forearm was $83.3^{\circ}$ (range: $70^{\circ}-90^{\circ}$ ), and the mean supination was $89.1^{\circ}$ (range: $75^{\circ}-90^{\circ}$ ) in group 0 ; the mean pronation was $81.3^{\circ}$ (range: $60^{\circ}-90^{\circ}$ ), the mean supination was $86.9^{\circ}$ (range: $45^{\circ}-90^{\circ}$ ) in group A. The mean grip strength was $81.6 \%$ (range: $38-91 \%$ ) in group $O$ and 80.2\% (range: $38-100 \%$ ) in group A (-Table 1).

\section{Postoperative Evaluation}

The final evaluation included pain, measurements of wrist and forearm motion, grip strength, ulnar head instability, the Disabilities of the Arm, Shoulder, and Hand (DASH) questionnaire, and the Mayo modified wrist score (MMWS). Postoperative pain was evaluated with a NRS, and preoperative pain was scored as 10 . Wrist flexion-extension was assessed with a goniometer (TTM-KO, Sakai Medical Co., Tokyo, Japan). Forearm supination and pronation were assessed with the elbow flexed $90^{\circ}$ at the patient's side. Grip strength was measured with a calibrated dynamometer (TKK 5401, Takei Machinery Co., Niigata, Japan) and reported as the ratio to the contralateral side. Ulnar head instability was examined with the ulnar head ballottement test and assessed with Nakamura et al's DRUJ instability score (0: no end point in any direction; 1: at least one endpoint either in dorsal or palmar; 2 : looser than the intact contralateral side; 4 : stable DRUJ). ${ }^{7}$

\section{Statistical Analysis}

The operating time, NRS score, range of motion (ROM), grasping power, the ulnar head instability score, and the DASH score for both procedures were compared using the $t$-test. The MMWS were compared with the chi-squared test. A $p$-value of $<0.05$ was regarded as significant.

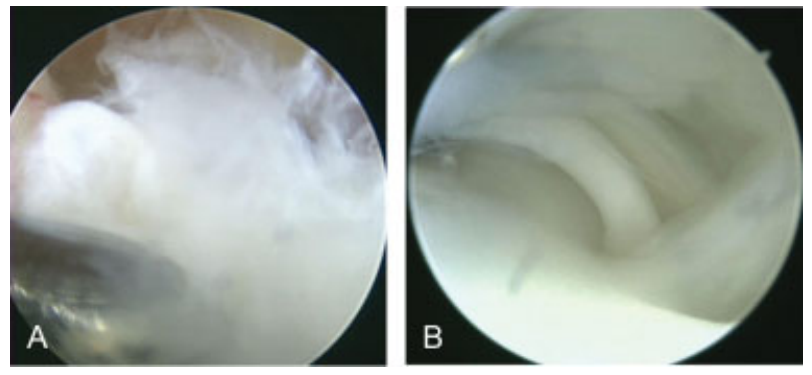

Fig. 1 Distal radioulnar joint (DRUJ) arthroscopy for the left wrist showed complete tear of the fovea (A). DRUJ arthroscopy for the right wrist represented the elongated ligament without tension (B).

\section{Surgical Technique}

The patient is placed in a supine position under general anesthesia with the affected arm on a hand table and a tourniquet placed on the proximal arm and inflated. The wrist is suspended in vertical traction and examined by arthroscopy. Generally, two dorsal arthroscopic portals are used: a 3-4 portal and a 4-5 portal to examine the RC joint. A 1.9 - or $2.3-\mathrm{mm}$ arthroscope with a $30^{\circ}$ angle is introduced through the 3-4 portal, and a probe, a shaver, and a radiofrequency device are interchangeably inserted through the 4-5 portal. The $6 \mathrm{U}$ portal is used as an outflow portal with the wet technique. If a foveal tear is present, TFCC tension becomes loose; therefore, loss of the trampoline effect is recognized. A peripheral tear (ulnar styloid tear ${ }^{10}$ ) of the TFCC should be also investigated through a hook test. Then, the TFCC foveal insertion is evaluated through the DRUJ portal. DRUJ arthroscopy can directly visualize a foveal tear (-Fig. 1A, B). The TFCC is thoroughly inspected through these portals.

Both open and arthroscopic repair are subsequently performed after diagnostic arthroscopy with keeping the forearm suspended by finger traps. In the open procedure, we recognized mild swelling at the surgical field after diagnostic wet arthroscopy; however, it did not affect the surgical approach so much. The open repair is started with about a $3-\mathrm{cm}$ straight skin incision on the ulnar side of the ulnar neck. The tendon sheath of the extensor carpi ulnaris (ECU) tendon is incised, and the ECU tendon is retracted palmarly or dorsally and freely mobilized. The ulnar wrist capsule is cut longitudinally, exposing the TFCC disc and the fovea under loupe magnification. A foveal lesion of the TFCC is recognized as a torn ligament or continuous scar tissue which is loose. The scar tissue should be debrided the minimum to confirm precise point of the attachment of the TFCC.

Two osseous tunnels are made by inserting two parallel 1.5-mm Kirschner wires (K-wire) from the ulnar neck to the foveal region. The point of K-wire insertion is the ulnar surface of the ulnar neck, 1.5 to $2.0 \mathrm{~cm}$ proximal from the distal end of the ulnar styloid. The place of bone tunnels is confirmed to be appropriate with image intensifier. The two sutures, 3-0 PDS (Ethicon, Somerville, NJ) and 3-0 Vicryl (Ethicon), are threaded horizontally at the ulnar peripheral lesion of the TFCC and pass through the two bone tunnels to 


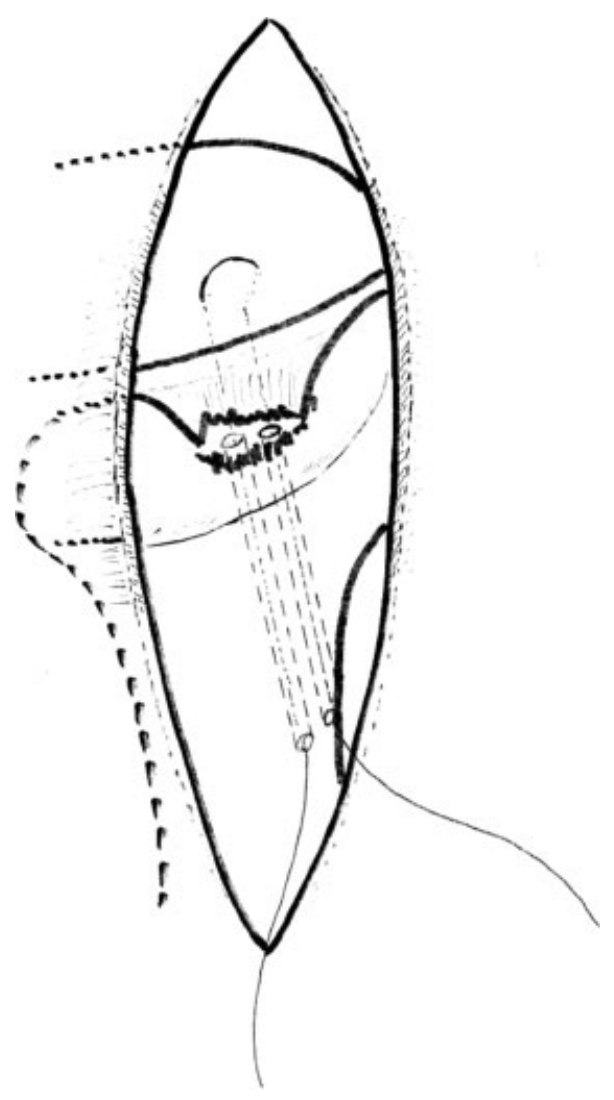

Fig. 2 The open approach. The suture passes through the two bone tunnels with open approach.

the ulnar surface of the ulnar neck (-Fig. 2). After loosening the traction of the forearm, and applying compression between the radius and ulna by the assistant in neutral forearm rotation, the threads were tied up with manual maximum tension to directly attach the TFCC to the fovea.

The arthroscopic repair is performed through a similar but shorter skin incision, and the ECU tendon is freely mobilized and expose the ulnar capsule. Two bone tunnels are created in a similar fashion with the direct repair. Two K-wires should be protruded within half to one-third ulnar side of TFCC, confirmed with RC arthroscopy. The location of the bone tunnels is also confirmed with an image intensifier. The two 21-gauge needles are inserted with a lasso loop of a 3-0 nylon suture through the two bone tunnels and the torn ulnar edge of the TFCC in the RC joint. The two looped sutures are retrieved through the $4-5$ portal using blunt mosquito forceps (-Fig. 3A), and then the two sutures, 3-0 PDS and 3-0 Vicryl, are threaded through the loop suture and introduced into the RC joint. Traction on the looped sutures then pulls the PDS and the Vicryl sutures through the TFCC and out through the two bone tunnels. The TFCC is tightly attached to the fovea with tying up the threads with manual maximum tension ( - Fig. 3B).

\section{Postoperative Management}

After repair, the postoperative protocol was consistent with both procedures. The wrist was fixed with a long-arm cast for 2 weeks with $90^{\circ}$ of elbow flexion and neutral forearm
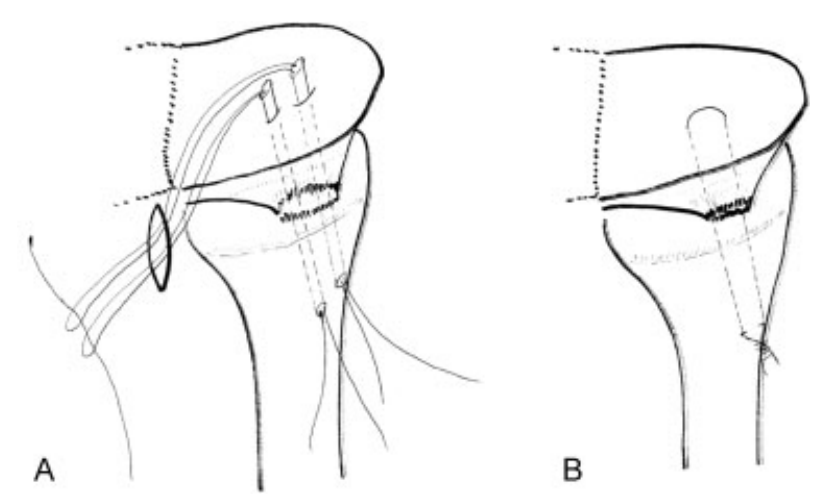

Fig. 3 The arthroscopic approach. The two loop sutures passed through the bone tunnels are retrieved through the 4-5 portal (A), the triangular fibrocartilage complex (TFCC) is tightly attached to the fovea with tying up the thread (B).

rotation. A short arm cast was applied for an additional 2 weeks. Gentle ROM exercise including rotation of the forearm was started at 4 weeks after surgery, and grip strengthening was started at 2 months. The patients were instructed that they could return to preoperative sports or work 3 to 6 months after surgery.

\section{Results}

All outcomes for both procedures are shown in - Table 2. The average operation time was 89.2 minutes (75-110 minutes) in group $\mathrm{O}$ and 55.3 minutes (30-80 minutes) in group $A$, significantly shorter than in group $\mathrm{O}(p=0.002)$. There was no patient who complained of wrist pain at the final followup in group 0 ; the average NRS was 0 . In group $A$, three patients felt mild ulnar-sided wrist pain during heavy activities. The average NRS was $0.2(0-2)$. The mean extension of the wrist was $66.6^{\circ}$ (range: $60^{\circ}-73^{\circ}$ ), and the mean flexion was $63.0^{\circ}$ (range: $50^{\circ}-70^{\circ}$ ) in group 0 ; the mean extension was $72.9^{\circ}$ (range: $60^{\circ}-85^{\circ}$ ), and the mean flexion was $66.3^{\circ}$ (range: $50^{\circ}-80^{\circ}$ ) in group $A$. The mean pronation of the forearm was $83.4^{\circ}$ (range: $80^{\circ}-90^{\circ}$ ), and the mean supination was $90.0^{\circ}$ (range: $85^{\circ}-95^{\circ}$ ) in group 0 ; the mean pronation was $83.3^{\circ}$ (range: $75^{\circ}-90^{\circ}$ ), and the mean supination was $89.4^{\circ}$ (range: $80^{\circ}-90^{\circ}$ ) in group A. The mean grip strength was $96.9 \%$ (range: $92-100 \%$ ) in group $O$ and $97.6 \%$ (range: $74-$ $115 \%$ ) in group A. DRUJ instability of all patients was evaluated as 4 in group $0 ; 18$ patients were assessed as 4 , and 3 patients were evaluated as 2 in group A, the average was 3.7. The mean DASH at final follow-up was $7.8(0-15.3)$ in group $\mathrm{O}$ and 5.7 (0-14.7) in group A. The final results according to the MMWS were all excellent in group 0 , with 18 excellent and 3 good in group $A$. There were no significant differences between the groups in the $t$-test $(p>0.05)$ and the chisquare test $(p>0.05)$ except for the operating time. There were no complications, and no patients needed reoperation in both groups. 
Table 2 Postoperative data in each group

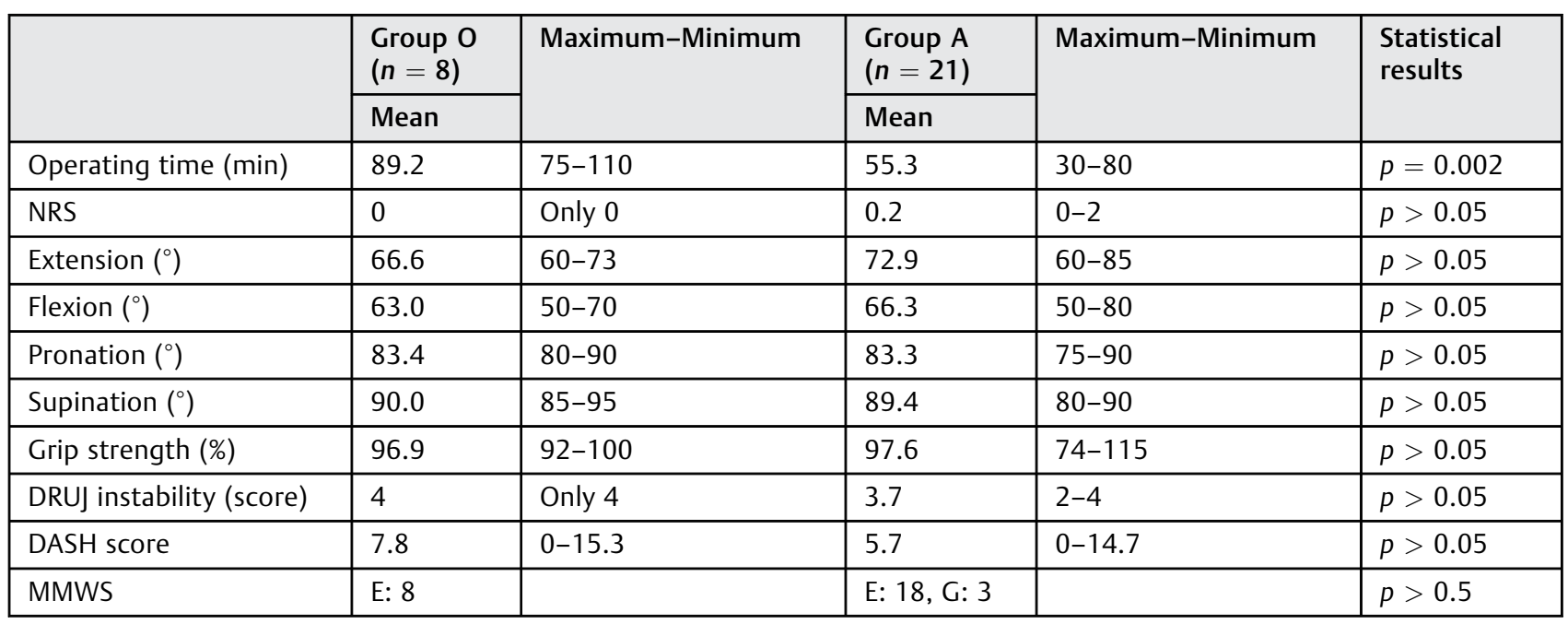

Abbreviations: DASH score, Disabilities of the Arm, Shoulder and Hand score; DRUJ, distal radioulnar joint; E, excellent; G, good; MMWS, Mayo modified wrist score; NRS, numerical rating scale.

Note: There were no significant differences between the two groups except for the operating time.

\section{Discussion}

In this study, we clarify that transosseous repair for TFCC foveal tear through both open and arthroscopic approach could provide feasible results. These results suggested that reattachment of the TFCC to the precise location, which was confirmed with macroscopy, arthroscopy, and image intensifier, is the critical point to reconstruct the TFCC foveal tear using the open or arthroscopic procedures. This investigation revealed that the ulnar attachment of the TFCC is divided into two sections: attachment to the ulnar styloid and the fovea. The tear on the ulnar side of TFCC inevitably occurs at these attachments. Nakamura and Makita described the detailed three-dimensional structure of the TFCC. During forearm rotation, the dorsal and volar portions of the distal radioulnar ligament show a nearly isometric length pattern at the fovea. ${ }^{3}$ Atzei has analyzed Palmer's $1 \mathrm{~B}$ tear $^{11}$ in detail and classified it into a distal component, a proximal component, or both, suggesting that fundamentally these tears can be repaired. ${ }^{9}$ Abe et al reported various patterns of TFCC tears and distinguished a foveal tear from an ulnar styloid tear. ${ }^{10}$

The foveal tear should be diagnosed precisely. The patient complains of a slack sensation during forearm rotation and loses strong grasping. A positive fovea sign is suggestive but not specific, because this sign may suggest not only a foveal tear, but a tear from the ulnar styloid and the inflammation at the surrounding structure. The ulnar head ballottement test is a reliable physical test. It must be evaluated bilaterally; the instability is more evident if it is examined under general anesthesia. MRI can delineate a foveal detachment clearly. A gradient echo sequence T2-weighted image provides a highdelineation image of the TFCC structure. However, evaluation of MRI findings is sometimes confusing when the ligament is continuous with scar tissue like in this study. DRUJ arthroscopy is a definitive procedure to diagnose a foveal tear. DRUJ arthroscopy is still a technically demanding procedure because the joint space is very narrow. However, when a foveal tear exists, the foveal region can easily be visualized through DRUJ arthroscopy, because the DRUJ is loose. The quality of the remnant fibers should be evaluated. If the remnant fibers are severely disrupted, primary repair is not indicated.

Several procedures for open and arthroscopic repair have been described. Moritomo described the open repair through a volar approach with the concept that foveal detachment would initially occur from the volar element. ${ }^{12}$ Atzei ${ }^{9}$ and Kim et al ${ }^{13}$ described a hybrid approach in which they explored the foveal lesion arthroscopically and used an open technique to reattach the foveal insertion using a bone anchor. Iwasaki et al described arthroscopic reattachment by creating an osseous tunnel, $2.9 \mathrm{~mm}$ in diameter, from the ulnar neck to the foveal surface, and their 2- to 4 -year follow-up results were good. ${ }^{14}$ Nakamura et al reported a three-dimensional mattress suture technique that can create an anatomical reconstruction using an open ulnar approach. ${ }^{15}$ Nakamura et al also described arthroscopic transosseous repair using their original targeting device. Their comparative study between the open and arthroscopic approaches showed that both procedures could obtain excellent clinical results. ${ }^{7}$ Shinohara et al performed arthroscopically assisted foveal repair primarily in accordance with the method of Nakamura, and they showed satisfactory outcomes with a mean follow-up of 30 months. ${ }^{16}$

There have been few reports of comparative studies between open and arthroscopic approaches. Anderson et al stated that there was no significant difference in clinical outcome after open versus arthroscopic repair. ${ }^{5}$ However, in this report, TFCC tear was classified as a 1B tear with Palmer's classification, and it was unclear whether the tear was a foveal tear or avulsion from the ulnar styloid. Arthroscopic repair was not described precisely. What procedure did the outside-in repair they mentioned mean; the capsular repair for avulsion 
from the ulnar styloid or transosseous repair for foveal tear? Luchetti et al reported successful outcomes with open and arthroscopically-assisted repairs. ${ }^{6}$ They confirmed the foveal detachment through DRUJ arthroscopy and repaired it using a suture anchor. They showed no significant postoperative differences between the two groups except for the DASH, which was significantly better in the arthroscopic group.

Our procedure that TFCC was reattached to the fovea by two threads pull-out technique through two bone tunnels in both approaches was totally different from previous comparative reports except for Nakamura et al's report. They obtained excellent clinical results in both procedures. ${ }^{7}$ However, they pointed out that the clinical results were unsatisfactory when arthroscopic repair was performed at an average of 19 months after injury. They also stated that the patients with $+2 \mathrm{~mm}$ positive ulnar variance resulted in unsatisfactory results when arthroscopic repair was performed. In this study, we obtained satisfactory results in all cases. This is because the patients with an ulnar positive variance of more than $+1 \mathrm{~mm}$ were excluded, and the mean duration of symptoms before surgery was 7.1 months. Shinohara et al stated that a patient with a traumatic foveal tear without ulnar abutment may be a good candidate for arthroscopic foveal repair even 7 months after the injury, and the present clinical results may have proven that. In addition, foveal repair was not indicated if DRUJ arthroscopy showed the unrepairable remnant of the ligament. DRUJ arthroscopy is essential to determine the indication for foveal repair.

The operating time was significantly shorter in the arthroscopic approach than in the open approach. This is because, in the open approach, we need to find the precise location of the fovea under loupe magnification. Reverse L-incision to the capsule may be suitable to expose the TFCC disc and the foveal lesion widely. However, we set the longitudinal incision to capsule not to open the RC and DRUJ joint widely. This procedure may be one of the reasons that open procedure took longer time than the arthroscopic approach because visual field was very narrow. In any case, threading the suture horizontally at the critical point of the TFCC through the open approach was a tough procedure.

\section{Limitations}

This study has several limitations. The number of cases was small, especially the number treated with the open approach. The indication for each procedure was determined by the time period of surgery; first, 8 patients were repaired with open approach and the following 21 patients were repaired arthroscopically. All surgeries were consecutive, and the open repairs were all conducted sequentially before the author converted to an arthroscopic approach. This is a potential significant source of bias of surgical skill. All surgery was performed by a single surgeon, and these cases were subject to the learning curve. The surgeon's ability to perform both the open and arthroscopic approach, especially the DRUJ arthroscopy, and suture naturally is fundamentally linked to the learning curve. That may affect the difference of the operating time.

\section{Conclusion}

Satisfactory outcomes were achieved in both the open repair and arthroscopic repair groups with strict indications. If a surgeon becomes familiar with the arthroscopic technique, it might be quicker and save operating time.

Note

This clinical investigation was conducted with the approval of our institutional review board.

Conflict of Interest

None.

\section{References}

1 af Ekenstam F, Hagert CG. Anatomical studies on the geometry and stability of the distal radio ulnar joint. Scand J Plast Reconstr Surg 1985;19(01):17-25

2 Nakamura T, Yabe Y, Horiuchi Y. Functional anatomy of the triangular fibrocartilage complex. J Hand Surg [Br] 1996;21(05):581-586

3 Nakamura T, Makita A. The proximal ligamentous component of the triangular fibrocartilage complex. J Hand Surg [Br] 2000;25 (05):479-486

4 Haugstvedt JR, Berger RA, Nakamura T, Neale P, Berglund L, An KN. Relative contributions of the ulnar attachments of the triangular fibrocartilage complex to the dynamic stability of the distal radioulnar joint. J Hand Surg Am 2006;31(03):445-451

5 Anderson ML, Larson AN, Moran SL, Cooney WP, Amrami KK, Berger RA. Clinical comparison of arthroscopic versus open repair of triangular fibrocartilage complex tears. J Hand Surg Am 2008; 33(05):675-682

6 Luchetti R, Atzei A, Cozzolino R, Fairplay T, Badur N. Comparison between open and arthroscopic-assisted foveal triangular fibrocartilage complex repair for post-traumatic distal radio-ulnar joint instability. J Hand Surg Eur Vol 2014;39(08):845-855

7 Nakamura T, Sato K, Okazaki M, Toyama Y, Ikegami H. Repair of foveal detachment of the triangular fibrocartilage complex: open and arthroscopic transosseous techniques. Hand Clin 2011;27 (03):281-290

8 Tay SC, Tomita K, Berger RA. The "ulnar fovea sign" for defining ulnar wrist pain: an analysis of sensitivity and specificity. J Hand Surg Am 2007;32(04):438-444

9 Atzei A. New trends in arthroscopic management of type 1-B TFCC injuries with DRUJ instability. J Hand Surg Eur Vol 2009;34(05): 582-591

10 Abe Y, Tominaga Y, Yoshida K. Various patterns of traumatic triangular fibrocartilage complex tear. Hand Surg 2012;17(02):191-198

11 Palmer AK. Triangular fibrocartilage complex lesions: a classification. J Hand Surg Am 1989;14(04):594-606

12 Moritomo H. Open repair of the triangular fibrocartilage complex from palmar aspect. J Wrist Surg 2015;4(01):2-8

$13 \mathrm{Kim}$ B, Yoon HK, Nho JH, et al. Arthroscopically assisted reconstruction of triangular fibrocartilage complex foveal avulsion in the ulnar variance-positive patient. Arthroscopy 2013;29(11):1762-1768

14 Iwasaki N, Nishida K, Motomiya M, Funakoshi T, Minami A. Arthroscopic-assisted repair of avulsed triangular fibrocartilage complex to the fovea of the ulnar head: a 2- to 4-year follow-up study. Arthroscopy 2011;27(10):1371-1378

15 Nakamura T, Nakao Y, Ikegami H, Sato K, Takayama S. Open repair of the ulnar disruption of the triangular fibrocartilage complex with double three-dimensional mattress suturing technique. Tech Hand Up Extrem Surg 2004;8(02):116-123

16 Shinohara T, Tatebe M, Okui N, Yamamoto M, Kurimoto S, Hirata $\mathrm{H}$. Arthroscopically assisted repair of triangular fibrocartilage complex foveal tears. J Hand Surg Am 2013;38(02):271-277 